\title{
ESTUDOS SOBRE POLÍTICAS PÚBLLICAS DE ESPORTE E LAZER DE PREFEITURAS MUNICIPAIS DO ESTADO DE SÃO PAULO
}

\author{
Antonia Dalla Pria Bankoff, Universidade Estadual de Campinas - UNICAMP, Campinas, \\ São Paulo - Brasil \\ Carlos Aparecido Zamai, Universidade Estadual de Campinas - UNICAMP, Campinas, São \\ Paulo - Brasil
}

\begin{abstract}
RESUMO
O objetivo deste foi realizar levantamento de dados sobre políticas públicas de esporte e lazer em municípios do Estado de São Paulo, tendo como base a Pesquisa Esporte, Turismo, Lazer, Atividade Física e Saúde na Cidade. Para a obtenção do perfil dos municípios em relação a este tema, foi elaborado um questionário contendo 14 perguntas abertas e fechadas, o qual foi enviado às prefeituras de 645 municípios do Estado de São Paulo via on line criado especificamente para esta pesquisa e enviado para as Prefeituras através de serviços de e-mail. Os índices atingidos em número de municípios que contribuíram com a pesquisa foram de $17,98 \%$ o que equivale a 106 prefeituras. Os resultados mostraram que os municípios estudados não possuem política definida sobre Políticas Públicas de Esporte e Lazer para a população, especificamente em relação a definição de espaços públicos; ginásios de esportes cobertos, programas de atividades físicas para a terceira idade e programas específicos para portadores de necessidades especiais. Constatou-se também, uma quantidade reduzida de projetos em municípios de até 10 mil habitantes havendo necessidade de maior apoio governamental e melhores definições sobre políticas públicas de esporte e lazer por parte da Secretaria de Estado de Esporte, Lazer e Turismo do Estado de São Paulo, evidenciando-se desta forma a necessidade de conscientização junto às prefeituras.
\end{abstract}

Palavras-Chave: Esportes; Lazer; Atividade Física; Projetos; Municípios.

\section{STUDIES ON PUBLIC POLICIES OF SPORTS AND LEISURE IN MUNICIPAL GOVERNMENTS OF THE STATE OF SÃO PAULO}

\begin{abstract}
In this study, we aimed at performing data collection on policies for sport and leisure in municipalities of the State of São Paulo, including as research basis tourism, leisure, physical activity, and health in the city. To obtain a profile of the municipalities for this subject, we developed a questionnaire containing 14 open and closed questions. We sent such questionnaire, created specifically for this study, to the local authorities of 645 municipalities of the State of São Paulo via e-mail. The rates obtained for the municipalities that contributed to the survey were $17.98 \%$, which equates to 106 local authorities. The results showed that the municipalities have no policy defined for Public Policies on Sport and Leisure for the
\end{abstract}


population, specifically concerning the definition of public spaces; gymnasia with roof; physical activity programmes for older people and specific programmes for people with special needs. We also verified a reduced number of projects in municipalities up to 10000 inhabitants with the need for greater government support and better definitions for public policies on sport and leisure by the State Department on Sport, Leisure, and Tourism of the State of São Paulo. Such fact evidences that the local authorities need to be aware of it.

Key-Words: Sports; Leisure; Physical activity; Projects; Municipalities. 


\section{INTRODUÇÃO}

Uma das principais características de toda a vida animal é o movimento, independente de seu tamanho e complexidade, que é gerado a todo o momento na busca pela sobrevivência, ora através de simples divisão celular, ora numa corrida de um predador à caça. O homem, para alimentação e proteção, acabou se movimentando intensamente por aproximadamente dois milhões de anos. ${ }^{1}$

Mas surgiram as máquinas, as maravilhas tecnológicas, a praticidade, e o homem foi parando, parando, parando... O grande trabalho físico que o homem realizava manualmente se torna tarefa executada por máquinas, onde o homem do final do milênio acaba por desempenhar tarefas que exigem pouco esforço físico. ${ }^{2}$

A discussão do tema políticas públicas tem recebido contínuas contribuições de pesquisadores das áreas de Ciências Sociais, Ciência Política, Serviço Social, dentre outras. Muito embora se admita a existência de atenção ao estudo do tema, há que se ressaltar que diferentes áreas dedicam-se a promover debates como trabalho e economia, podendo desembocar, inclusive, para setores como saúde e educação, mas não se projeta para o esporte e lazer. . $^{3-4}$

As discussões sobre políticas públicas deverão atingir todos os segmentos sociais e a periferia da cidade, buscando a distribuição democrática de recursos existentes com prioridade dos recursos públicos para recreação e lazer, incorporando à dinâmica da cidade, as práticas corporais e esportivas como direito assegurado de afirmação cultural e de cidadania. ${ }^{5}$

Conexões: revista da Faculdade de Educação Física da UNICAMP, Campinas, v. 9, n. 2, p.70-84, maio/ago. 2011. ISSN: 1983-9030. 
De um lado um avanço tecnológico que possibilitou um aumento da longevidade da espécie humana, no tocante a evolução do tratamento e na prevenção de doenças infecto-contagiosas, principal causa de morbidade e mortalidade anterior a década de 30. De outro, um avanço gradativo, comparando-se dados epidemiológicos atuais com os do século passado, do índice de morbidade e mortalidade decorrentes de doenças crônicas degenerativas, que pode hoje ser considerado como epidemia de final de século. ${ }^{1}$

Quanto à temática do lazer é hoje reconhecida no campo acadêmico nacional e internacional. No Brasil temos grupos de estudos, pesquisadores, revistas especializadas e programas de pósgraduação que elaboram significativas reflexões sobre a questão. No entanto, ainda é necessário estreitar as relações do campo acadêmico com as instituições que colocam em prática políticas para o lazer, especialmente as de ordem pública. ${ }^{6}$

O tema das políticas públicas de esporte e lazer instiga estudiosos de diferentes áreas de interesse, sendo há anos fonte de investigação para diversas pesquisas. ${ }^{1,7}$ No campo das iniciativas governamentais, especialmente a partir da década de 1980, podemos identificar diferentes ações que visam garantir o esporte e o lazer como direitos sociais à população. De acordo com Pinto ${ }^{8}$, “a garantia dos direitos sociais, buscando reduzir progressivamente as desigualdades, passa a se constituir como investimento assegurado pelo Estado, pelo setor estatal, não governamental e também pelo setor corporativo". No entanto, apesar das iniciativas em prol de assegurar aos cidadãos brasileiros o direito ao esporte e ao lazer, ainda permanecem barreiras sociais, econômicas e culturais que dificultam este processo. ${ }^{6}$

Conexões: revista da Faculdade de Educação Física da UNICAMP, Campinas, v. 9, n. 2, p.70-84, maio/ago. 2011. ISSN: 1983-9030. 
A Educação Física escolar, numa importante investida de descaracterização de todo e qualquer vinculo com a aptidão física atlética, acabou por deixar de lado, infelizmente, objetivos como habilitar os alunos a compreenderem os determinantes fisiológicos, biomecânicos, sóciopolítico-econômicos e culturais da prática desportiva e da atividade física em geral, deixando de contribuir para construção de estilos de vida mais saudáveis e de um maior conhecimento do binômio exercício-saúde. ${ }^{9}$

Assim, nos grandes centros urbanos, a possibilidade da prática de atividade física, lazer e esportes se vinculam a existência de academias e clubes que oferecem esses serviços, porém observa-se um acesso limitado por parte de toda a população, decorrentes da dimensão do espaço físico ou pelos custos financeiros que representam.

Diante desta e de outras investigações, está bem claro a insuficiência de locais e de apoio por parte das prefeituras na implementação de programas de atividades físicas, lazer e esportes junto da população para melhor qualidade de vida, acreditando que as prefeituras podem proporcionar a população, mediante implementação de políticas públicas para construção e criação de locais para a prática de atividades físicas, lazer e esporte.

\section{OBJETIVO GERAL}

O objetivo deste foi realizar um levantamento de dados sobre políticas públicas de esporte e lazer das prefeituras municipais do Estado de São Paulo.

Conexões: revista da Faculdade de Educação Física da UNICAMP, Campinas, v. 9, n. 2, p.70-84, maio/ago. 2011. ISSN: 1983-9030. 


\section{MATERIAL E MÉTODO}

\section{Material Utilizado}

Foi elaborado um questionário contendo 14 perguntas abertas e fechadas, abordando diversas questões sobre esporte, lazer, turismo e programas de atividades físicas voltados à saúde da população no Estado de São Paulo. O trabalho foi desenvolvido pelo Núcleo de Educação a Distância em Exercício Físico e Saúde da Faculdade de Educação Física - UnicampCampinas/SP, no período de agosto de 2008 a agosto de 2009.

\section{DESENVOLVIMENTO DA PESQUISA}

Inicialmente, foi desenvolvido um banco de dados através de pesquisa on line e dos e-mails das prefeituras, estabelecendo-se contactos com o chefe do gabinete das prefeituras dos municípios do Estado de São Paulo, sobre o objetivo e a importância da pesquisa, também como acessar o questionário da mesma via on line. Após estes contatos, através de um link criado especificamente para esta pesquisa foi enviado o referido questionário por e-mail para as 645 prefeituras, estabelecendo-se um prazo de trinta dias após o envio para efetuarem as respostas.

Após este procedimento, foi disponibilizado um número de telefone e uma pessoa treinada para tirar as dúvidas e colaborar no que fosse preciso em relação à orientação do questionário. Vencido o prazo estabelecido de 30 dias, reenviamos todo material e aguardamos por mais trinta dias para as prefeituras responderem o mesmo, totalizando 60 dias corridos. 
De posse dos questionários respondidos pelas Prefeituras Municipais, iniciou-se o processo de tabulação e análise dos resultados. Portanto, neste trabalho serão apresentados os resultados de 07 questões do questionário aplicado nos municípios.

\section{Questões}

Quadro 1 - Apresentação das 7 questões selecionadas do questionário

\begin{tabular}{cl}
\hline Questões & Perguntas \\
\hline Q1 & Quantas praças de esporte ou centros esportivos existem no município? \\
Q2 & Quantos ginásios de esportes existem no município? Q2a Público e Q2b Privados? \\
Q3 & Quantas escolinhas de iniciação esportiva existem no município? Q3a Pública e Q3b Privada? \\
Q4 & Quantos parques públicos existem no município para o desenvolvimento de lazer? \\
Q5 & Quantos clubes sociais para atividades esportivas existem no município? \\
Q6 & Há atividades (Programas) específicas para a terceira idade? \\
Q7 & Há atividades (Programas) específicas para portadores de deficientes? \\
\hline
\end{tabular}

\section{RESULTADOS}

A tabulação dos questionários respondidos e devolvidos pelas prefeituras, estão apresentados em tabelas para melhor entendimento, onde foi considerado uma divisão de 05 grupos por número de habitantes.

Quadro 2 - Divisão dos grupos e total de habitantes acumulados por grupo

\begin{tabular}{lrr}
\hline GRUPOS & TOTAL/HABITANTES & $\begin{array}{r}\text { VALOR } \\
\text { ACUMULADO }\end{array}$ \\
\hline Grupo A & $0-10.000$ habitantes & 226.346 \\
Grupo B & $10.001-30.000$ habitantes & 461.989 \\
Grupo C & $30.001-50.000$ habitantes & 395.999 \\
Grupo D & $50.001-100.000$ habitantes & 908.228 \\
Grupo E & Acima de 100.000 habitantes & 5.576 .225 \\
\hline
\end{tabular}

Conexões: revista da Faculdade de Educação Física da UNICAMP, Campinas, v. 9, n. 2, p.70-84, maio/ago. 2011. ISSN: 1983-9030. 
Desta forma, os resultados apresentados a seguir se referem aos dados coletados em 106 municípios distribuídos conforme a Tabela 1.

Tabela 1 - Distribuição dos dados coletados em 44 municípios de 0 a 10 mil habitantes, 25 municípios de 10.001 a 30 mil habitantes, 09 municípios de 30.001 a 50 mil habitantes, 13 municípios de 50.001 a 100 mil habitantes e 15 municípios acima de 100mil habitantes referentes às políticas públicas de esporte e lazer do Estado de São Paulo.

\begin{tabular}{cccccc}
\hline Perguntas & $\begin{array}{c}\text { Municípios } \\
\text { até 10 mil } \\
\text { habitantes }\end{array}$ & $\begin{array}{c}\text { Municípios } \\
\text { até 30 mil } \\
\text { habitantes }\end{array}$ & $\begin{array}{c}\text { Municípios } \\
\text { até 50 mil } \\
\text { habitantes }\end{array}$ & $\begin{array}{c}\text { Municípios } \\
\text { até 100 mil } \\
\text { habitantes }\end{array}$ & $\begin{array}{c}\text { Municípios } \\
\text { acima de 100 } \\
\text { mil habitantes }\end{array}$ \\
\hline Q1 & 12 & 26 & 46 & 41 & Q \\
Q2 & Q2a P 24 & Q2a P 22 & Q2a P13 Q2b & Q2a P 69 & Q2a P 75 \\
& Q2b PR 02 & Q2b PR 08 & PR10 & Q2b PR 25 & Q2b PR 185 \\
Q3 & Q3a P 31 & Q3a P 42 & Q3a P 10 & Q3a P 112 & Q3a P 341 \\
& Q3b PR 10 & Q3b PR 32 & Q3b PR 19 & Q3b PR 61 & Q3b PR 193 \\
Q4 & 22 & 22 & 49 & 25 & 83 \\
Q5 & 25 & 17 & 18 & 55 & 179 \\
Q6 & 06 & 08 & 5 & 12 & 16 \\
Q7 & 01 & 06 & 5 & 9 & 15 \\
\hline
\end{tabular}

Legenda: $\mathbf{P}=$ público, $\mathbf{P R}=$ privado.

Tabela 2 - Distribuição dos dados coletados em 106 municípios referentes às proporções de espaços públicos e particulares para o desenvolvimento de políticas públicas de esporte e lazer do Estado de São Paulo por habitante.

\begin{tabular}{cccccc}
\hline Perguntas & $\begin{array}{c}\text { Municípios } \\
\text { até 10 mil } \\
\text { habitantes }\end{array}$ & $\begin{array}{c}\text { Municípios até } \\
\text { 30 mil } \\
\text { habitantes }\end{array}$ & $\begin{array}{c}\text { Municípios até } \\
\mathbf{5 0} \text { mil } \\
\text { habitantes }\end{array}$ & $\begin{array}{c}\text { Municípios até } \\
\text { 100 mil } \\
\text { habitantes }\end{array}$ & $\begin{array}{c}\text { Municípios } \\
\text { acima de 100 } \\
\text { mil habitantes }\end{array}$ \\
\hline Q1 & 18.862 & 17.768 & 8.608 & 22.151 & 17.590 \\
Q2a & 9.431 & 20.999 & 30.461 & 13.162 & 74.349 \\
Q2b & 113.173 & 57.748 & 39.599 & 36.329 & 30.141 \\
Q4 & 10.288 & 20.999 & 8.081 & 36.329 & 67.183 \\
Q5 & 9.053 & 27.175 & 21.999 & 16.513 & 31.152 \\
Q6 & 37.724 & 57.748 & 79.199 & 75.685 & 348.514 \\
\hline
\end{tabular}

Conexões: revista da Faculdade de Educação Física da UNICAMP, Campinas, v. 9, n. 2, p.70-84, maio/ago. 2011. ISSN: 1983-9030. 


\section{DISCUSSÃO}

O índice atingido quanto ao número de questionários respondidos e devolvido ao Núcleo de Educação a Distância em Exercício Físico e Saúde - FEF-UNICAMP foi de 17,98\% do total de municípios, o que equivale a 106 prefeituras do Estado de São Paulo. O prazo estipulado para realizarem a resposta foi inicialmente 30 dias. Após este período, estipulamos mais trinta dias com o objetivo de aumentar o número de respostas pelas prefeituras. Reconhecemos que o percentual de resposta foi relativamente baixo $(17,98 \%)$.

É interessante observar que a maior concentração dos municípios que mostraram interesse em participar da pesquisa através de resposta do questionário ficou alocada no $1^{\circ}$ grupo, ou seja, até 10 mil habitantes, sendo 44 municípios, mesmo porque deve ser esta a realidade, ou seja, a maior parte dos municípios do Estado de São Paulo se encontra nesta faixa de população. As dificuldades para se obter as respostas através das prefeituras é um fator importante na condução e desenvolvimento desta pesquisa, impedindo assim, um melhor mapeamento, sobre políticas de esporte e lazer do Estado de São Paulo, sendo a intenção da mesma.

As Tabelas 1 e 2 mostram os resultados coletados onde observamos os municípios que responderam a questão 01 possuem praças de esportes ou centros esportivos proporcionalmente, não muito diferentes entre a classificação dos municípios, com destaque para os municípios de até 50 mil habitantes, onde a proporção de habitantes para ocupação destes espaços é menor comparando com a classificação de municípios.

Conexões: revista da Faculdade de Educação Física da UNICAMP, Campinas, v. 9, n. 2, p.70-84, maio/ago. 2011. ISSN: 1983-9030. 
Em relação aos ginásios de esporte existentes nas cidades (públicos e privados) os resultados apontados na Tabela 1 mostraram que os municípios de até 10 mil habitantes possuem maior número de ginásio público do que privado e a proporção por habitante quanto ao público, mostrado na Tabela 2 é menor quando comparado com as outras classificações por habitantes, porém, quanto aos ginásios privados o resultado mostrou insuficiência para os municípios de até 10 mil habitantes. Nos municípios acima de 100 mil habitantes é maior e existência de ginásios de esportes privados. A Tabela 2 ainda mostra que à medida que os municípios aumentam as populações, a proporção de pessoas com acesso aos ginásios de esportes públicos também aumentam. A condição parece mais confortável nesta questão para os municípios até 100 mil habitantes.

Quanto às escolas de iniciação esportiva (Questão 3) elas são em número maior no setor público, independente da classificação dos grupos de habitantes. Com isso, parece-nos que os municípios se fazem presentes para cumprir seu papel quanto ao desenvolvimento de iniciação esportiva oportunizando as crianças vivenciarem o aprendizado em modalidades esportivas. Nos municípios de até 50 mil habitantes predominou as empresas privadas o desenvolvimento das escolas de iniciação esportivas. É interessante observar que nos municípios de até 10 mil habitantes muitos deles não possuem escola de iniciação esportiva pública por município (31 escolas de iniciação esportiva para 44 municípios). A presença de escolinhas de esportes nos municípios é muito importante para o desenvolvimento e crescimento do esporte no Brasil no que se refere ao desenvolvimento do cidadão, bem como, na formação de futuros atletas de competição nas modalidades esportivas em nível nacional e internacional.

Conexões: revista da Faculdade de Educação Física da UNICAMP, Campinas, v. 9, n. 2, p.70-84, maio/ago. 2011. ISSN: 1983-9030. 
Em suas colocações Pinto, ${ }^{7}$ discorre sobre "a garantia dos direitos sociais, buscando reduzir progressivamente as desigualdades, passa a se constituir como investimento assegurado pelo Estado, pelo setor estatal não governamental e também pelo setor corporativo". No entanto, apesar das iniciativas em prol de assegurar aos cidadãos brasileiros o direito ao esporte e ao lazer, ainda permanecem barreiras sociais, econômicas e culturais que dificultam este processo. ${ }^{8}$ Os autores citados relatam os direitos assegurados pelos cidadãos brasileiros ao esporte e lazer, porém, reconhecem as dificuldades e as barreiras sociais.

A Questão de número 4 pergunta quantos parques públicos existem na cidade para a prática de lazer. Esta pergunta através dos resultados na Tabela 1 demonstra o quanto os municípios deixam a desejar com a implementação de política públicas do lazer, especificamente aos parques públicos. Esta pesquisa mostra que os municípios independentes do número de habitantes não investem em políticas públicas de esporte e lazer, refletindo na qualidade de vida do cidadão, uma vez que ele possui poucas opções de lazer em sua cidade. Isso deve contribuir com o aumento de pessoas cada vez mais dentro de suas casas e também acreditamos na presença maior de pessoas em locais privados como, por exemplo, em bares, shopping, locais inadequados para lazer e outros. Com isso, leva-se a crer que a população de nível sócio-econômico inferior é a que mais sofre as consequiências por falta de organização de espaços públicos para o desenvolvimento de políticas públicas de esporte e lazer.

Se observarmos a Tabela 2 pode-se verificar que os municípios acima de 150 mil habitantes a proporção de número de pessoas para cada parque público é extremamente alto, ou seja, está 
em torno de 67 mil habitantes, comprometendo assim a qualidade de vida da população destes municípios em busca do lazer.

A presença dos clubes sociais nas cidades, conforme retrata a questão de número 5 , os que dependem dos chamados "sócios", a pesquisa mostrou que nem todos os municípios possuem clubes sociais que se destinam as práticas e o desenvolvimento de políticas públicas de lazer. A Tabela 2 demonstra a proporção de habitantes por clube. Os clubes sociais conforme mostra a pesquisa on line demonstram serem locais frequientados por pessoas que contribuem com valores pagos mensalmente, o que dificulta a classe social de nível sócio-econômico inferior participar destes locais.

Um dado apontado na Tabela 1 em relação à Questão 6, quanto a existência de programas específicos para a terceira idade nos município, verificamos que a quantidade ofertada a população é muito inferior ao esperado, uma vez que o número de pessoas da terceira idade e mais tarde idosos tem aumentado progressivamente em nosso país. Estes dados demonstram que os governos municipais não estão preocupados com esta causa, ou seja, maior investimento em programas de políticas públicas de esportes e lazer destinado às pessoas da terceira idade e idosos. Talvez seja por isso, que no Brasil temos tantos "Asilos e Casas destinadas ao cuidar do Idoso".

Ainda em relação à Questão 6, onde os municípios acima de 150 mil habitantes mostram que a cada 348.514 pessoas os municípios ofertam apenas um programa de atividade física para a terceira idade e para o idoso, verificamos através do $\mathrm{IBGE}^{10}$ uma das cidades que respondeu o 
questionário, ela apresenta uma população de 145.328 habitantes para as faixas etárias de 65 a 86 anos e não oferta nenhum programa para a terceira idade e idoso.

Outro fator importante foi abordado na questão de número 7, quanto aos programas de atividades físicas específicas para os portadores de necessidades especiais. Os resultados demonstrados na Tabela 1 não retratam a preocupação quanto à qualidade de vida dos sujeitos portadores de necessidades especiais. Isso relata a mais pura realidade brasileira, especificamente a do Estado de São Paulo o quanto os governos municipais não investem em projetos para esta população. Nos municípios de até 10 mil habitantes praticamente não existem programas de políticas públicas para o desenvolvimento do esporte e lazer para os portadores de necessidades especiais.

\section{CONSIDERAÇÕES FINAIS}

Enquanto os governos Estadual e Municipal do Estado de São Paulo se preocupem com falas supérfluas a população sofre as consequiências da falta de políticas públicas para o desenvolvimento do esporte e do lazer, contribuindo desta maneira com o desenvolvimento do sedentarismo, o crescimento das doenças crônicas não transmissíveis e uma qualidade de vida mais propensa ao estresse.

Há necessidade de maiores investimento através do Governo Estadual para com as Prefeituras no que diz respeito às Políticas Públicas de Esporte e Lazer e também maior reflexão sobre o cuidar da população, criando núcleos regionais de debates sobre estas temáticas na tentativa de 
melhorar a qualidade de vida da população, especificamente as de nível socioeconômico inferior.

\section{REFERÊNCIAS}

${ }^{1}$ BANKOFF, A. D. P.; ZAMAI, C. A.; SILVA, J. F. da. et al. Concepções sobre a prática de atividades físicas e saúde na educação física e esportes. Conexões: revista da Faculdade de Educação Física da UNICAMP, Campinas, v. 7, n. 3, p. 86-102, 2009.

${ }^{2}$ FRANCISCHETTI, A. C. Trabalho sedentário: um problema para a saúde do trabalhador. Campinas: Ed. da Unicamp, 1990.

${ }^{3}$ BANKOFF, A. D. P. et al. Estudo do perfil de municípios do Estado de São Paulo sobre espaços físicos e projetos voltados à saúde da população. Movimento e Percepção, Espírito Santo do Pinhal, v. 5, n. 7, 2005.

${ }^{4}$ SUASSUNA, D. M. F. A. Políticas públicas para o esporte e o lazer no Brasil (19962005). Disponível em: http://observatoriodoesporte.org.br/politicas-publicas-para-o-esporte-eo-lazer-no-brasil-1996-2005/ Acesso em: 11 out. 2010.

${ }^{5}$ BRUST, C.; BAGGIO, I. C.; SALDANHA FILHO, M. F. Gestão das políticas públicas de esporte e lazer em Santa Maria, RS. Efdeportes, Buenos Aires, ano 12, n. 116, 2008. Disponível em: http://www.efdeportes.com/efd116/gestao-das-politicas-publicas-de-esporte-elazer.htm 8/10/10. Acesso em: 11 set. 2008.

Conexões: revista da Faculdade de Educação Física da UNICAMP, Campinas, v. 9, n. 2, p.70-84, maio/ago. 2011. ISSN: 1983-9030. 
${ }^{6}$ MENDES, R.; CUNHA JUNIOR, C. F. F. da. Compreensões sobre o lazer. Licere, Belo Horizonte, v. 12, n. 3, set. 2009.

${ }^{7}$ BANKOFF, A. D. P.; ZAMAI, C. A.; SILVA, J. F. da. Estudos sobre políticas públicas de esporte e lazer de prefeituras municipais do Estado de São Paulo. In: ENCUENTRO INTERNACIONAL DE LA ASSOCIACION LATINOAMERICANA DE ESTUDIOS SOCIOCULTURALES DEL DEPORTE, 2., 2010, Maracay. Anais... Maracay, 2010.

${ }^{8}$ PINTO, L. M. S. de M. Políticas públicas de lazer no Brasil: uma história a contar. In: MARCEllinO, N. C. (Org.). Políticas públicas de lazer. Campinas: Alínea, 2008.

${ }^{9}$ GUEDES, D. P.; GUEDES, J. E. R. P. Exercício físico na promoção da saúde. Londrina: Midiograf, 1995.

${ }^{10}$ INSTITUTO BRASILEIRO DE GEOGRAFIA E ESTATÍSTICA (IBGE). Perfil dos municípios brasileiros: gestão pública: 2001. Disponível em: HTTP://www.ibge.gov.br/home/estatistica/economia/perfilmunic/2001/default.shtm. Acesso em: 12 out. 2010 .

Esta pesquisa teve apoio financeiro do: CNPq - Processo $n^{\circ}$ 520006/96-8 e FAPESP Processo no $1996 / 5708-4$. 